\title{
Endovaskuläre Therapie des akut ischämischen Schlaganfalls unter Analgosedierung im Vergleich zur Intubationsnarkose - Durchführbarkeit, periprozedurale Sicherheit, klinisches und radiologisches Outcome
}

\author{
Endovascular Treatment of Acute Ischemic Stroke Under Conscious Sedation Compared to General Anesthesia - \\ Safety, Feasibility and Clinical and Radiological Outcome
}

Autoren

Institute
S. Langner ${ }^{1}$, A. V. Khaw ${ }^{2}$, T. Fretwurst ${ }^{1}$, A. Angermaier ${ }^{2}$, N. Hosten ${ }^{1}$, M. Kirsch ${ }^{1}$

Institut für Diagnostische Radiologie und Neuroradiologie, Universitätsmedizin Greifswald

Klinik und Poliklinik für Neurologie, Universitätsmedizin Greifswald

\author{
Key words \\ - brain \\ - thrombolysis \\ - ischemia/infarction
}

eingereicht 17.4 .2012

akzeptiert 9.11 .2012

Bibliografie

DOI http://dx.doi.org/

10.1055/s-0032-1330361

Online-Publikation: 18.2.2013

Fortschr Röntgenstr 2013; 185: 320-327 @ Georg Thieme

Verlag KG Stuttgart · New York . ISSN 1438-9029

Korrespondenzadresse PD Dr. Sönke Langner Institut für Diagnostische Radiologie und Neuroradiologie, Universitätsmedizin Greifswald Ferdinand-Sauerbruch-Str. 1 17475 Greifswald

Tel.: $++49 / 3834 / 866960$

Fax: $++49 / 3834 / 867097$

langso@uni-greifswald.de

\section{Zusammenfassung}

$\nabla$

Ziel: Ein entscheidender Faktor in der Therapie des akuten ischämischen Schlaganfalls ist die Rekanalisation des Gefäßverschlusses. Endovaskuläre Therapieverfahren spielen eine immer bedeutendere Rolle. Es besteht kein Konsens, ob hierfür eine Intubationsnarkose (ITN) notwendig ist oder die lokale Anästhesie (LA) im Zugangsbereich und bedarfsweise Analogosedierung ausreichend sind. Ziel der Studie war es, die Durchführbarkeit der lokalen Schlaganfalltherapie unter LA zu evaluieren.

Material und Methoden: Über 5 Jahren wurden retrospektiv 131 Patienten mit einem akut ischämischen Schlaganfall eingeschlossen, die endovaskulär behandelt wurden. Rekanalisationsrate, primäres anästhesiologisches Vorgehen, Konversion von LA zu ITN, Infarktvolumen sowie Komplikationen wurden systematisch analysiert.

Ergebnisse: Bei 7 Patienten kam es technisch bedingt zu einem Therapieabbruch, 65 (52\%) der verbliebenen 124 Patienten (mittleres Alter 68,8 $\pm 14,6$ ) waren weiblich. Der Verschluss lag bei 94 (76\%) bzw. 30 Patienten (24\%) im vorderen bzw. im hinteren Stromgebiet. 105 Patienten (85\%) wurden in LA, $16(13 \%)$ primär in ITN behandelt. In 3 Fällen (2\%) erfolgte eine Konversion von LA in ITN. Eine primäre Thrombolyse bzw. mechanische Rekanalisation erfolge bei 60 (48\%) bzw. 27 (22\%) Patienten, in 37 Fällen (30\%) erfolgte eine Kombinationstherapie. Zwischen der LA- und ITN-Gruppe bestand weder hinsichtlich Rekanalisationsrate noch Komplikationen ein statistisch signifikanter Unterschied. Die Interventions- und Rekanalisationsdauer war in LA signifikant kürzer $(\mathrm{p}<0,01)$.

Schlussfolgerung: Endovaskuläre rekanalisierende Verfahren in der Therapie des akuten ischämischen Schlaganfalls sind unter LA mit niedrigem Komplikationsrisiko möglich und gehen mit einer kürzeren Interventionsdauer einher.

\section{Abstract \\ $\nabla$}

Purpose: Vessel recanalization is an important predictor of clinical outcome in the treatment of acute ischemic stroke. Endovascular therapies are used with increasing frequency. There is no general agreement on the policy of anesthesia during endovascular therapy, ranging from general anesthesia (GA) to local anesthesia at the puncture site with conscious sedation (CS) as needed. The aim of the study was to evaluate the safety and feasibility and radiological and clinical outcome of endovascular stroke therapy under CS.

Materials and Methods: We retrospectively included all patients with acute ischemic stroke who were treated with endovascular therapy over a five-year period. Data was evaluated with respect to type of sedation, conversion from CS to GA, recanalization rate, infarct volume and peri- and postprocedural complications.

Results: There was a technical failure in 7 patients. Of the remaining 124 patients (mean age 68.8 \pm 14.6 years), 65 were female ( $52 \%$ ). The site of occlusion was located in the anterior circulation in 94 patients $(76 \%)$ and in the posterior circulation in 30 cases (24\%). 105 patients (85\%) were treated under CS and 16 cases (13\%) primarily under GA. In 3 cases ( $2 \%$ ) peri-procedural conversion to GA was necessary. Primary intra-arterial thrombolysis, mechanical recanalization only, and combination therapy were performed in 60 (48\%), 27 $(22 \%)$ and $37(30 \%)$ patients, respectively. There were no significant differences for recanalization rate and complications between GA and CS. The mean procedure time was significantly shorter in patients treated under CS $(\mathrm{p}<0.01)$.

Conclusion: Endovascular stroke therapy with CS is feasible, can be performed safely and is faster than with GA. 


\section{Einleitung}

Für Patienten mit einem akuten ischämischen Schlaganfall stellt die frühe Rekanalisation des betroffenen Gefäßes einen entscheidenden prognostischen Faktor dar [1]. Seit der Veröffentlichung der ECASS-III-Studie (European Cooperative Acute Stroke Study) 2008 liegt Evidenz höchster Klasse für die Wirksamkeit der intravenösen Thrombolyse (ivT) im erweiterten Zeitfenster bis $4,5 \mathrm{~h}$ vor [2]. Jedoch liegt die Rekanalisationsrate der ivT unter denen der lokalen intra-arteriellen Thrombolyse (iaT) und der Erfolg der ivT sinkt zusätzlich mit der Größe des betroffenen Gefäßes [1, 3]. Daher finden lokal rekanalisierende Verfahren bei der Behandlung verschlossener großer intrakranieller Gefäße eine immer stärkere Verbreitung [4-8].

Prinzipiell kann eine solche Intervention in Intubationsnarkose (ITN) oder unter lokaler Anästhesie und bedarfsweiser Analgosedierung (LA) durchgeführt werden [9-12]. Mit dem in der Regel schweren neurologischen Defizit gehen häufig eine psychomotorische Unruhe und Unkooperativität einher. Vorteil der ITN ist, dass unkontrollierte Patientenbewegungen in viel geringerem Maße auftreten, welche das Risiko periprozeduraler Komplikationen erhöhen können [13, 14]. Demgegenüber geht die LA ohne eine Zeitverzögerung einher und der neurologische Status des Patienten kann zu jedem Zeitpunkt periinterventionell und während der Prozedur beurteilt werden [10].

Obwohl das Augenmerk meist auf das Rekanalisationsergebnis der Intervention als entscheidender Faktor für das klinische Outcome gerichtet ist, bleibt die Frage des adäquaten Patientenmanagements während der Intervention ungeklärt und variiert zwischen verschiedenen neurointerventionellen Zentren [9-12]. In unserem Zentrum werden Patienten für eine solche Intervention routinemäßig nicht intubiert, um eine Zeitverzögerung bis zum Beginn der Intervention zu vermeiden. Bislang liegen keine kontrollierten Daten zum Einfluss des anästhesiologischen Vorgehens auf das neurologische Outcome vor, die eine evidenzbasierte Empfehlung gestatten.

Ziel der vorliegenden retrospektiven Studie war es, in einem Patientenkollektiv die Durchführbarkeit und periprozedurale Sicherheit lokal rekanalisierender Verfahren bei einem akut ischämischen Schlaganfall unter LA gegenüber der ITN zu evaluieren.

\section{Material und Methoden}

$\nabla$

\section{Einschlusskriterien}

In die Studie wurden Patienten eingeschlossen, die zwischen Juli 2005 und Juli 2010 in unserer Abteilung aufgrund eines akuten ischämischen Schlaganfalls interventionell behandelt wurden. Weitere Einschlusskriterien waren ein moderates bis schweres neurologisches Defizit (entsprechend $>4$ Punkte im National Institute of Health Stroke Scale [NIHSS]) sowie Abschluss der Diagnostik und Beginn der Intervention innerhalb von $6 \mathrm{~h}$ nach Symptombeginn bei Insulten des vorderen Stromgebiets. Patienten mit einem Infarkt im vertebrobasilären Stromgebiet wurden in die Studie eingeschlossen, solange sich in der Diagnostik kein Infarkt demarkierte, unabhängig vom Zeitpunkt seit Symptombeginn.

\section{Präinterventionelle Diagnostik}

Entsprechend der Leitlinien der Stroke Unit unseres Hauses erhielten alle Patienten mit dem klinischen Verdacht auf einen akuten Schlaganfall initial ein natives CCT. Zeigte dieses keine Blu- tung und betrugen die Infarktfrühzeichen [15] weniger als ein Drittel des Media-Stromgebiets, so erfolgte die Durchführung einer CT-Angiografie (CTA) und einer CT-Perfusion (CTP). Die CTA umfasste die kraniozervikalen Gefäße von Höhe HWK 6 bis zum Vertex. Bei einem Gefäßverschluss im vorderen Kreislauf erfolgte die Durchführung der CTP auf Höhe der Basalganglien, bei einer vertebrobasilären Okklusion wurden die Schichten für die CTP so eingelegt, dass unter Aussparung der Linse Pons und beide Kleinhirnhemisphären erfasst wurden.

Bei einer Verschlusslokalisation im Bereich der extra- und intrakraniellen Arteria carotis interna (ACI), der Carotis-Bifurkation (Carotis-T), der Arteria cerebri media (ACM) im M1- und M2-Segment sowie bei Verschlüssen der Arteria basilaris erfolgte im interdisziplinären Konsens zwischen Neurologie und Neuroradiologie der Entschluss zur endovaskulären Therapie.

\section{Interventionelle Prozedur}

Patienten mit einer Kontraindikation für eine medikamentöse Thrombolyse [16] wurden im gesamten Studienzeitraum primär mittels mechanischer Thrombektomie behandelt. Ansonsten erfolgte primär eine medikamentöse Thrombolyse. Kam es hierunter nach 20 mg rt-PA (Actilyse ${ }^{\circledR}$, Boehringer, Deutschland) zu keiner Reduktion der Thrombuslast, erfolgte die Eskalation der Therapie und der Wechsel zur mechanischen Thrombektomie.

Alle Interventionen erfolgten an einer biplanaren AngiografieEinheit (Integris IV, Philips Healthcare, Niederlande) über einen transfemoralen 6F-Zugang. Der Führungskatheter wurde bei Verschlüssen im vorderen Stromgebiet in der ipsilateralen ACI unterhalb der Schädelbasis, ansonsten in der dominanten Arteria vertebralis platziert. In koaxialer Technik erfolgte die Sondierung des verschlossenen Gefäßes mit dem Mikrokatheter. Die Auswahl des verwendeten Führungskatheters, Mikrodrahts sowie -katheters oblag dem interventionellen Neuroradiologen. Im Zeitraum von Juli 2005 bis Dezember 2006 war die lokale Thrombolyse mittels rt-PA (Actilyse, Boehringer, Deutschland) die einzige interventionelle Therapieoption.

Der Mikrokatheter wurde hierfür in dem proximalen Thrombus platziert und das Thrombolytikum mit $1 \mathrm{mg} / \mathrm{min}$ bis zur vollständigen Rekanalisation bzw. bis zu einer Maximaldosis von 0,9 mg/ kg Körpergewicht appliziert. Die korrekte Lage des Mikrokatheters bzw. der Grad der Rekanalisation wurde angiografisch alle 10 min überprüft.

Die mechanische Rekanalisation erfolgt seit Januar 2007 bzw. April 2008 mit dem Phenox Clotretriever PCR und CRC, seit Januar 2010 mit dem BONnet-System (Phenox GmbH, Bochum, Deutschland) oder seit Juli 2009 mit dem Penumbra-System (Penumbra Europe, Berlin, Deutschland). Auch hierfür oblag die Auswahl des Thrombektomiesystems dem interventionellen Neuroradiologen. Das Ausmaß der Rekanalisation wurde nach jedem Rekanalisationsversuch überprüft. Bei Verwendung des Penumbra-Systems wurde der Aspirationskatheter unmittelbar proximal des Thrombus platziert und die Thrombusaspiration unter Verwendung der „Road map“-Technik durchgeführt. Rekanalisationsergebnis und die korrekte Katheterlage wurden angiografisch alle 5 min überprüft.

Als intraprozedurale Komplikationen galten eine Perforation oder Dissektion des verschlossenen Gefäßes bzw. im Zugangsbereich. Das Ausmaß der Rekanalisation wurde in der letzten Serie abschließend entsprechend der TICI-Kriterien [17] beurteilt. Der Zeitraum zwischen Beginn der Diagnostik und dem Zeitpunkt des letzten Angiogramms wurde als „door to recanalization time“ definiert. Zusätzlich wurde für alle Patienten die Dauer 
der Intervention von Beginn der arteriellen Punktion bis zum letzten Angiogramm dokumentiert.

\section{Patientenmanagement}

Periinterventionell erfolgt die Überwachung der Patienten mittels EKG, Pulsoxymetrie sowie nicht invasiver Blutdruckmessung. Alle nicht intubierten Patienten erhielten Sauerstoff mit 2 1/min über eine Nasensonde und die Intervention erfolgte in Anwesenheit eines Neurologen mit intensivmedizinischer Erfahrung sowie eines Anästhesisten. Nicht intubierten Patienten wurde der Kopf mittels Lagerungshilfen fixiert. Bei mangelnder Compliance erfolgte die bedarfsweise Analgosedierung mit Midazolam (Dormicum, Roche Pharma AG, Schweiz) oder Diazepam (Valium ${ }^{\circledR}$, Roche Pharma AG, Schweiz) und Dipidolor (Piritramid ${ }^{\circledR}$, Janssen-Cilag, Belgien). Hierfür wurden sowohl Dipidolor als auch das Benzodiazepin intravenös titriert. Initial erfolgte die Gabe eines $3 \mathrm{mg}$ Bolus des Benzodiazepins und anschließend die Analgosedierung in 1mg-Schritten die Gabe von Piritramid und in 2mg-Schritten für das Benzodiazepin unter Monitoring der klinischen Vigilanz bis zum erkennbaren Sistieren der psychomotorischen Unruhe und Müdigkeit bei erhaltener prompter Reaktion auf Ansprache.

Postinterventionell erfolgte bei nicht intubierten Patienten die Verlegung auf die Stroke Unit für mindesten $24 \mathrm{~h}$. Intubierte Patienten wurden postinterventionell auf die Intensivstation verlegt und ein zeitnaher Aufwach- und Extubationsversuch durchgeführt. Nach erfolgreicher Extubation erfolgte die Verlegung auf die Stroke Unit.

\section{Auswertung}

Der neurologische Status der Patienten wurde mittels NIHSS bei Aufnahme, unmittelbar postinterventionell, $24 \mathrm{~h}$ nach Intervention sowie bei Verlegung von der Stroke Unit auf die „post Stroke“-Station oder eine Rehabilitationseinrichtung dokumentiert. Ein Kontroll-CCT zur Beurteilung der Infarktdemarkierung sowie dem Auftreten von Blutungskomplikationen entsprechend der ECASS-II-Kriterien [16] erfolgte $24 \mathrm{~h}$ nach Intervention, nach 5 Tagen sowie bei neurologischer Verschlechterung um mehr als 2 Punkte im NIHSS. Das finale Infarktvolumen wurde im Kontroll-CCT nach 5 Tagen mittels Volumen-Tool der CT-Konsole (SIEMENS Medical Systems, Erlangen, Deutschland) bestimmt.

Zusätzlich diente bei allen Patienten als Surrogatparameter für die Schwere des Schlaganfalls die Größe des Infarktkerns in der CBV(zerebrales Blutvolumen)-Parameterkarte (Schwellenwert $<13 \mathrm{ml} / 100 \mathrm{ml}$ ) [18 - 20] sowie die maximale Größe der Perfusionsstörung in der TTP(time to peak)-Parameterkarte (Schwellenwert $>150 \%$ im Vergleich zur Gegenseite) in der CTP [21]. Hierfür wurde in den Schichten der CTP um das entsprechende Areal eine manuelle Region-of-Interest (ROI) eingelegt. Zur Beurteilung des Therapieerfolgs wurde in den korrespondierenden Schichten des finalen CCT die Größe des Infarktareals im Verhältnis zur Perfusionsstörung in der TTP bestimmt.

Im weiteren Verlauf wurden die Patienten hinsichtlich Aufenthaltsdauer auf der Intensivstation sowie Stroke Unit sowie dem Auftreten periinterventioneller Komplikationen evaluiert. Als periinterventionelle Komplikationen galten eine intrakranielle Blutung, rekurrenter Hirninfarkt, Myokardinfarkt, das Auftreten einer Pneumonie während des Aufenthalts auf der Stroke Unit sowie Komplikationen im Bereich des arteriellen Zugangs mit Ausbildung eines Leistenhämatoms, welches eine operative Therapie oder eine Transfusion erforderte, oder eines Pseudoaneurysmas.

\section{Statistische Analyse}

Die eingeschlossenen Patienten wurden in 3 Gruppen eingeteilt. Bei Patienten in Gruppe I erfolgte die Intervention in Lokalanästhesie im Zugangsbereich und bedarfsweiser Analgosedierung (LA). Gruppe II umfasste die Patienten, bei denen während der Intervention eine Intubation erfolgen musste und Gruppe III die Patienten, die primär intubiert zur Diagnostik und Intervention kamen. Alle quantitativen Parameter werden als Mittelwert \pm Standardabweichung dargestellt. Die statistische Analyse erfolgte mittels des Mann-Whitney-U-Tests sowie des $\mathrm{Chi}^{2}$-Tests unter Verwendung von SPSS Vers. 16. Ein p-Wert $<0,05$ galt als signifikant.

Die Datenerhebung bezüglich des Krankheitsverlaufs erfolgte retrospektiv nach Abschluss der Studie durch Auswertung der elektronischen Patientenakten, der Dokumentation der Stroke Unit und der digital verfügbaren Röntgenbilder.

Vor Durchführung der Studie lag ein positives Votum der zuständigen Ethikkommission vor.

\section{Ergebnisse \\ $\nabla$}

Im Studienzeitraum wurden 131 Patienten eingeschlossen. Aus technischen Gründen wurde die Intervention bei 7 Patienten abgebrochen, da aufgrund ausgeprägter Gefäßelongation der Mikrokatheter weder im betroffenen Gefäß noch im Thrombus selbst platziert werden konnte. Diese Patienten gehörten der Gruppe I an und wurden aus der weiteren statistischen Analyse ausgeschlossen, sodass für die finale Auswertung 124 Patienten zur Verfügung standen. Von diesen Patienten wurden 86 (69\%) innerhalb des 3-hFensters behandelt, 35 (28\%) zwischen 3 und 6 h nach Symptombeginn. Bei 3 Patienten (2\%) kam es zu einer Protokollverletzung, da sie außerhalb des 6-h-Zeitfensters behandelt wurden. Dies erfolgt in einem Fall aufgrund des jungen Alters des Patienten (25 Jahre) und in 2 Fällen aufgrund der anamnestisch angegebenen präklinischen Gesamtsituation sowie der fehlenden Infarktfrühzeichen im nativen CCT. Gruppe I umfasste 105 Patienten (85\%), Gruppe II und III 3 bzw. 16 Patienten (2 bzw. 13\%).

Zwischen Patienten mit einem Verschluss im vorderen und hinteren Kreislauf bestand statistisch kein signifikanter Unterschied hinsichtlich Alters- und Geschlechtsverteilung. Ebenso bestand innerhalb der Gruppe I kein signifikanter Unterschied für den NIHSS bei Aufnahme zwischen den beiden Verschlusslokalisationen ( $\bullet$ Tab.1). Da sich in den Gruppen II und III nur sehr geringe Patientenzahlen mit einem Gefäßverschluss im anterioren und im posterioren Kreislauf fanden, wurden diese für die jeweilige Verschlusslokalisation zusammengefasst. Da die Gruppe II/III jedoch signifikant häufiger Patienten mit einem Verschluss im vertebrobasilären Stromgebiet enthielt als Gruppe I (12 von 30 Patienten [40\%] vs. 7 von 94 Patienten [7\%] im vorderen Kreislauf; $\mathrm{p}<0,001$ ), erfolgt die weitere Betrachtung der Ergebnisse unter Berücksichtigung der Verschlusslokalisation. Im gesamten Studienkollektiv wurden 60 (48\%) mittels alleiniger iaT behandelt, 27 (22\%) mittels alleiniger mechanischer Rekanalisation und 37 (30\%) mit einer Kombinationstherapie. Die Verteilung der einzelnen Verfahren unterschied sich nicht signifikant zwischen LA und ITN.

\section{Verschluss im vorderen Kreislauf}

Im vorderen Kreislauf wurden 51 Patienten (54\%) mittels alleiniger iaT, 24 (26\%) rein mechanisch rekanalisiert, und in 26 Fällen (28\%) erfolgte eine Kombinationstherapie. Die mittlere Interventionsdauer war in Gruppe I signifikant kürzer (102 \pm 37 min vs. 
Tab. 1 Demografische Daten.

\begin{tabular}{|c|c|c|c|c|c|c|}
\hline Demografie & gesamt & Gruppe I & Gruppe II/III & p-Wert & Gruppe II & Gruppe III \\
\hline $\mathrm{n}($ in \%) & 124 & $105(85)$ & $19(15)$ & & 3 & 16 \\
\hline Geschlecht (w/m) & $65 / 59$ & $59 / 46$ & $6 / 13$ & 0,048 & $0 / 3$ & $6 / 10$ \\
\hline vorderer Kreislauf (w/m) & $53 / 41$ & $50 / 37$ & $3 / 4$ & n.b. & $0 / 2$ & $3 / 2$ \\
\hline \multirow[t]{2}{*}{ hinterer Kreislauf (w/m) } & $12 / 18$ & $9 / 9$ & $3 / 9$ & n. b. & $0 / 1$ & $3 / 8$ \\
\hline & gesamt & Gruppe I & Gruppe II/III & p-Wert & & \\
\hline \multirow[t]{2}{*}{ Alter ${ }^{2}$} & $68,7 \pm 14,6$ & $69,7 \pm 14,1$ & $63,3 \pm 16,3$ & 0,11 & $62 \pm 16,8$ & $63,5 \pm 16,8$ \\
\hline & $17-97 ; 72$ & $17-97 ; 73$ & $25-81 ; 67$ & & $49-81 ; 56$ & $25-81 ; 68,5$ \\
\hline Frauen & $70,6 \pm 14,2$ & $71,3 \pm 13,6$ & $63,2 \pm 18,7$ & 0,18 & & $\begin{array}{l}73,5 \pm 8,4 \\
63-88 ; 72,5\end{array}$ \\
\hline Männer & $66,7 \pm 14,9$ & $67,7 \pm 14,6$ & $63,3 \pm 15,2$ & 0,55 & $\begin{array}{l}62 \pm 16,8 \\
49-81 ; 56\end{array}$ & $\begin{array}{l}60,3 \pm 19,4 \\
25-81 ; 67\end{array}$ \\
\hline vorderer Kreislauf & $\begin{array}{l}71 \pm 12,7 \\
25-95 ; 73\end{array}$ & & & & & \\
\hline \multirow[t]{2}{*}{ hinterer Kreislauf } & $\begin{array}{l}62,9 \pm 17,8 \\
17-95 ; 66\end{array}$ & $\begin{array}{l}60,1 \pm 19,6 \\
17-95 ; 64\end{array}$ & $\begin{array}{l}63,1 \pm 15,8 \\
26-81 ; 67\end{array}$ & 0,581 & & \\
\hline & gesamt & Gruppe I & Gruppe II/III & p-Wert & & \\
\hline \multicolumn{7}{|l|}{ Verschlusslokalisation (n;\%) } \\
\hline vorderer Kreislauf & $94(76)$ & $87(83)$ & $7(37)$ & 0,22 & $2(67)$ & $5(31)$ \\
\hline $\mathrm{ACl}$ & $1(1)$ & 0 & $1(5)$ & & & $1(6)$ \\
\hline Carotis-T & $15(12)$ & $13(12)$ & $2(10)$ & & & $2(13)$ \\
\hline M1 & $55(44)$ & $52(50)$ & $3(16)$ & & $1(33)$ & $2(13)$ \\
\hline M1 / M2 & $23(19)$ & $22(21)$ & $1(5)$ & & $1(33)$ & \\
\hline hinterer Kreislauf & $30(24)$ & $18(17)$ & $12(63)$ & $<0,001$ & $1(33)$ & $11(69)$ \\
\hline Basilaris-Kopf & $9(7)$ & $8(8)$ & $1(5)$ & & & $1(6)$ \\
\hline Basilaris & $21(17)$ & $10(10)$ & $11(58)$ & & $1(33)$ & $10(62)$ \\
\hline A. vertebralis & 0 & 0 & 0 & & & \\
\hline \multicolumn{7}{|l|}{ NIHSS (Aufnahme) ${ }^{3}$} \\
\hline vorderer Kreislauf & $\begin{array}{l}14,1 \pm 5,3 \\
5-24 ; 14\end{array}$ & $\begin{array}{l}14,1 \pm 5,3 \\
5-24 ; 14,5\end{array}$ & & & $\begin{array}{l}14 \pm 1,4 \\
13-15 ; 14\end{array}$ & \\
\hline hinterer Kreislauf & $\begin{array}{l}16,9 \pm 7,3 \\
7-24 ; 16\end{array}$ & $\begin{array}{l}16,9 \pm 7,3 \\
7-24 ; 16\end{array}$ & & & 15 & \\
\hline \multicolumn{7}{|l|}{ Infarktgröße $\left(\mathrm{cm}^{2}\right)^{2}$} \\
\hline Fläche CBV & $145,3 \pm 72,4$ & $139,8 \pm 66,9$ & $142,8 \pm 78,3$ & 0,56 & $144,4 \pm 76,5$ & $141,1 \pm 80,2$ \\
\hline vorderer Kreislauf & $271,1 \pm 90,3$ & $261,2 \pm 106,4$ & $265,8 \pm 126,7$ & 0,57 & $270,9 \pm 83,4$ & $261,1 \pm 88,7$ \\
\hline hinterer Kreislauf & $19,3 \pm 28,6$ & $18,7 \pm 27,1$ & $19,8 \pm 29,9$ & 0,53 & 18,5 & $21,1 \pm 29,8$ \\
\hline Fläche TTP & $194,5 \pm 98,6$ & $197,3 \pm 102,7$ & $190,9 \pm 97,2$ & 0,43 & $191,6 \pm 93,3$ & $190,2 \pm 98,4$ \\
\hline vorderer Kreislauf & $363,4 \pm 67,9$ & $348,3 \pm 50,2$ & $382 \pm 52,2$ & 0,48 & $376,4 \pm 64,2$ & $389,4 \pm 68,2$ \\
\hline hinterer Kreislauf & $25,6 \pm 12,5$ & $22,4 \pm 16,8$ & $28,4 \pm 11,9$ & 0,38 & 27,5 & $29,4 \pm 13,5$ \\
\hline
\end{tabular}

1 n. b.: nicht berechenbar.

2 Mittelwert \pm Standardabweichung; zusätzlich für Alter und NIHSS Spannweite und Median.

${ }^{3}$ Gilt für Gruppe II, in Gruppe III aufgrund Intubation initialer NIHSS nicht möglich.

$134 \pm 69 \mathrm{~min}$ ), ebenso die „door to recanalization time“ (114 \pm 42 min vs. $194 \pm 49 \mathrm{~min}$ ). In Gruppe II/III kam es zu einer hämodynamisch nicht relevanten Dissektion der Arteria carotis interna (ACI). In 9 Fällen (10\%) war in Gruppe I eine bedarfsweise Analgosedierung notwendig. Davon lag der Verschluss in 6 Fällen in der sprachdominanten Hemisphäre mit konsekutiver schwerer Aphasie. Die Konversion zur ITN erfolgte in beiden Fällen der Gruppe II aufgrund einer respiratorischen Erschöpfung, die in einem Fall auf eine kardiale Dekompensation zurückzuführen war und im zweiten Fall postiktal.

Der Lokalisation des Gefäßverschlusses entsprechend war das Ausmaß der CTP-Veränderungen deutlich größer als in der Patientengruppe mit einem Verschluss im hinteren Stromgebiet ( $\bullet$ Tab. 1), ein signifikanter Unterschied zwischen Gruppe I und II/III bestand jedoch nicht. Details der Intervention sind in $\bullet$ Tab. 2 dargestellt. Die mittlere Aufenthaltsdauer auf der ITS betrug für Patienten der Gruppe II 1,2 $\pm 0,8$ Tage, für Patienten der Gruppe III 1,3 $\pm 0,6$ Tage. Patienten der Gruppe I hatten eine signifikant kürzere Aufenthaltsdauer auf der Stroke Unit (6,5 $\pm 3,7$ Tage vs. 9,6 $\pm 4,1$ Tage; $\mathrm{p}<0,01)$. Zusätzlich waren in Gruppe I sowohl das finale Infarktvolumen $\left(115 \pm 33,9 \mathrm{~cm}^{3}\right.$ vs. $\left.155,6 \pm 22,9 \mathrm{~cm}^{3} ; \mathrm{p}<0,01\right)$ als auch das Verhältnis Infarktvolumen/TTP $(0,34 \pm 0,6$ vs. $0,47 \pm 0,8$; $\mathrm{p}=0,01$ ) signifikant niedriger. Bezüglich des Auftretens weiterer Komplikationen während des Aufenthalts im Krankenhaus unterschieden sich beide Gruppen nicht signifikant ( $\bullet$ Tab. 3).

\section{Verschluss im hinteren Kreislauf}

Ein Verschluss im vertebrobasilären Stromgebiet wurde bei 23 (77\%) mittels alleiniger iaT, bei 5 Patienten (17\%) mittels alleiniger mechanischer Thrombektomie und in 2 Fällen (7\%) mit einer kombinierten Therapie behandelt. Auch in dieser Subgruppe unterschied sich die Verteilung der einzelnen Verfahren zwischen den beiden anästhesiologischen Gruppen statistisch nicht signifikant. Wie im vorderen Stromgebiet waren bei Verschlüssen im hinteren Stromgebiet sowohl die Interventionsdauer ( $97 \pm 39$ min vs. 133 $\pm 57 \mathrm{~min})$ als auch die „door to recanalization time“ ( $125 \pm 34 \mathrm{~min}$ vs. $158 \pm 23 \mathrm{~min}$ ) in Gruppe I signifikant kürzer als in Gruppe II/III ( $\bullet$ Tab. 2). Anders als bei Verschlüssen in der anterioren Zirkula- 


\begin{tabular}{|c|c|c|c|c|}
\hline Therapieart & gesamt & Gruppe I & Gruppe II/III & p-Wert ${ }^{1}$ \\
\hline nur medikamentöse (n; in \%) & $60(48)^{2}$ & $51(85)$ & $9(15)$ & \\
\hline vorderer Kreislauf & 37 & 35 & 2 & \\
\hline hinterer Kreislauf & 23 & 16 & 7 & \\
\hline nur mechanisch (n; in \%) & $27(22)^{2}$ & $24(89)$ & $3(11)$ & \\
\hline PCR/CRC & $16(59,3)$ & $13(48,2)$ & $3(11)$ & \\
\hline BONnet & $2(7,4)$ & $2(7,4)$ & 0 & \\
\hline Penumbra & $9(33,3)$ & $9(33,3)$ & 0 & \\
\hline vorderer Kreislauf & 22 & 20 & 2 & \\
\hline hinterer Kreislauf & 5 & 2 & 3 & \\
\hline Kombinationstherapie ( $\mathrm{n}$; in \%) & $37(30)^{2}$ & $26(70)$ & $11(30)$ & \\
\hline PCR/CRC & $23(62,1)$ & $15(41)$ & $8(22)$ & \\
\hline BONnet & $8(21,6)$ & $7(19)$ & $1(3)$ & \\
\hline Penumbra & $6(16,1)$ & $4(11)$ & $2(5)$ & \\
\hline vorderer Kreislauf & 35 & 32 & 3 & \\
\hline hinterer Kreislauf & 2 & 0 & 2 & \\
\hline Therapieabbruch & 7 & 7 & 0 & \\
\hline vorderer Kreislauf & 7 & 7 & 0 & \\
\hline hinterer Kreislauf & 0 & 0 & 0 & \\
\hline Interventionsdauer (min) & $105 \pm 44$ & $99 \pm 38$ & $134 \pm 63$ & $<0,01$ \\
\hline vorderer Kreislauf & $106 \pm 47$ & $102 \pm 37$ & $134 \pm 69$ & $<0,01$ \\
\hline hinterer Kreislauf & $103 \pm 42$ & $96 \pm 39$ & $133 \pm 57$ & $<0,01$ \\
\hline “door to recanalization"-time (min) & $148 \pm 49$ & $127 \pm 39$ & $168 \pm 35$ & 0,02 \\
\hline vorderer Kreislauf & $154 \pm 37$ & $114 \pm 42$ & $194 \pm 49$ & $<0,01$ \\
\hline hinterer Kreislauf & $142 \pm 19$ & $126 \pm 34$ & $158 \pm 29$ & $<0,01$ \\
\hline \multicolumn{5}{|l|}{ Rekanalisationsgrad ( $\mathrm{n}$; in \%) ${ }^{3}$} \\
\hline $\mathrm{TICl} 0 / 1 / 2 \mathrm{a}$ & $51(41)^{2}$ & $43(41)$ & $8(42,1)$ & 0,93 \\
\hline vorderer Kreislauf & 46 & 41 & 5 & \\
\hline hinterer Kreislauf & 5 & 2 & 3 & \\
\hline $\mathrm{TICl} 2 \mathrm{~b} / 3$ & $73(59)^{2}$ & $62(59)$ & $11(57,9)$ & \\
\hline vorderer Kreislauf & 48 & 46 & 2 & \\
\hline hinterer Kreislauf & 25 & 16 & 9 & \\
\hline
\end{tabular}

Tab. 2 Interventionsdaten

Sofern kein Wert angegeben, ließ sich dieser aufgrund geringer Fallzahlen nicht berechnen.

${ }^{2}$ Bezogen auf die Gesamtstudienpopulation.

${ }^{3}$ Bezogen auf die jeweilige Subgruppengröße der Gruppe I und Gruppe II/III.

tion kam es zu keinen interventionsbedingten Komplikationen. In einem Fall erfolgte ebenfalls aufgrund einer respiratorischen Erschöpfung bei kardialer Dekompensation die Konversion von LA zu ITN. Die mittlere Aufenthaltsdauer auf der Stroke Unit war auch in diesem Kollektiv für Gruppe I signifikant kürzer als für Gruppe II/III (6,3 $\pm 2,9$ vs. 7,9 $\pm 3,7$ Tage; $p=0,03)$.

Im Vergleich zu Patienten mit einem Verschluss im vorderen Kreislauf traten im weiteren Verlauf häufiger Pneumonien auf (7 [23\%] vs. 11 [11\%]), allerdings nicht statistisch signifikant. Ebenso bestand für die Mortalität zwischen den beiden Verschlusslokalisationen kein signifikanter Unterschied $(p=0,131)$. Details des postinterventionellen Verlaufs sind in $\bullet$ Tab. 3 dargestellt.

\section{Diskussion \\ $\nabla$}

Das adäquate Patientenmanagement für die endovaskuläre Schlaganfalltherapie ist nicht einheitlich definiert und unterscheidet sich zwischen verschiedenen neurointerventionellen Zentren [5, 912]. Demnach ist auch in den aktuellen endovaskulären Schlaganfallstudien $[6,8,22,23]$ diesbezüglich auch keine Vorgehensweise festgelegt, sodass der Einflussfaktor des anästhesiologischen Managements zwischen den Studien nicht gut vergleichbar ist, sofern er überhaupt untersucht wurde.

In der vorliegenden Arbeit konnten wir zeigen, dass die interventionelle Schlaganfalltherapie - sowohl die iaT als auch mechanische Verfahren oder eine Kombinationstherapie - unter lokaler
Anästhesie und bedarfsgesteuerter Analgosedierung mit niedriger prozeduraler Komplikationsrate möglich ist. Im Vergleich zur Intervention in ITN ging sie in unserem Studienkollektiv sowohl bei einem Verschluss im vorderen als auch vertebrobasilären Stromgebiet jeweils mit einer kürzeren Interventionsdauer, „door to recanalization time“ sowie niedrigerer Verweildauer auf der Stroke Unit einher. Zusätzlich war bei beiden Verschlusslokalisationen bei Intervention in LA das finale Infarktvolumen im Kontroll-CCT im Verhältnis zur initialen Perfusionsstörung des jeweiligen Gefäßterritoriums kleiner.

Die Ergebnisse der vorliegenden Studie werden durch die Arbeit von Jumaa et al. gestützt [10]. In einer retrospektiven Studie von 126 Patienten mit Verschluss der Arteria cerebri media (ACM) im M1-Segment konnten sie ebenfalls mögliche Vorteile einer Analgosedierung bezüglich des klinischen Outcomes aufzeigen. Ebenso fanden Sugg et al. in einer retrospektiven Analyse ihrer Patienten aus der MERCI-Studie eine besseres Outcome nach Intervention in LA gegenüber der in ITN [12]. Wir fanden ähnliche Ergebnisse auch in der Subgruppe von Patienten mit einem Verschluss im vertebrobasilären Stromgebiet. Von 105 Patienten der Gruppe I lag der Verschluss in 18 Fällen (17\%, versus 12 Fälle [63\%, p<0,001] in Gruppe II/III) im vertebrobasilären Stromgebiet. Diese ungleiche Verteilung überrascht nicht aufgrund der bei Hirnstamminfarkten häufig vorliegenden Schluck- und Atemstörungen, die eine Intubation notwendig machen können. In 3 Fällen musste periinterventionell die Konversion zur ITN erfolgen, jedoch lag in 2 dieser Fälle der Verschluss im M1-Segment der ACM. Patienten mit einem Ver- 


\begin{tabular}{|c|c|c|c|c|}
\hline Blutungskomplikationen (n) & gesamt & Gruppe I & Gruppe II/III & p-Wert ${ }^{1}$ \\
\hline keine Blutung & 86 & 77 & 11 & \\
\hline Blutung & 36 & 28 & 8 & 0,17 \\
\hline Hämorrhagische Transformation & 15 & 11 & 4 & 0,15 \\
\hline parenchymale Blutung & 21 & 17 & 4 & 0,43 \\
\hline finales Infarktvolumen $\left(\mathrm{cm}^{3}\right)$ & $79,5 \pm 51,2$ & $64,3 \pm 43,1$ & $92,6 \pm 63,1$ & 0,02 \\
\hline vorderer Kreislauf & $\begin{array}{l}135,3 \\
\pm 62,3\end{array}$ & $\begin{array}{l}115,4 \\
\pm 33,9\end{array}$ & $155,6 \pm 22,9$ & $<0,01$ \\
\hline hinterer Kreislauf & $23,7 \pm 19,8$ & $18,3 \pm 11,3$ & 29,1 & 0,03 \\
\hline Verhältnis TTP vs. Infarkt & $0,34 \pm 0,6$ & $0,32 \pm 0,9$ & $0,48 \pm 0,5$ & $<0,01$ \\
\hline vorderer Kreislauf & $0,35 \pm 0,6$ & $0,34 \pm 0,6$ & $0,47 \pm 0,8$ & $<0,01$ \\
\hline hinterer Kreislauf & $0,30 \pm 0,55$ & $0,31 \pm 0,2$ & $0,38 \pm 0,4$ & $<0,01$ \\
\hline \multicolumn{5}{|l|}{ NIHSS } \\
\hline \multicolumn{5}{|l|}{ vorderer Kreislauf } \\
\hline 24 hp.i. & $11,4 \pm 6,0$ & $11,4 \pm 6,3$ & $11,7 \pm 4,5$ & 0,78 \\
\hline Verlegung aus SU & $8,0 \pm 5,2$ & $7,8 \pm 5,2$ & $9,4 \pm 5,3$ & 0,21 \\
\hline \multicolumn{5}{|l|}{ hinterer Kreislauf } \\
\hline 24 h p. i. & $12,3 \pm 5,9$ & $12,1 \pm 5,1$ & $12,9 \pm 6,7$ & 0,66 \\
\hline Verlegung aus SU & $8,3 \pm 4,7$ & $8,1 \pm 5,6$ & $8,5 \pm 2,3$ & 0,71 \\
\hline \multicolumn{5}{|l|}{ Aufenthaltsdauer Intensivstation (in Tagen) } \\
\hline vorderer Kreislauf & \multicolumn{2}{|l|}{$1,2 \pm 0,8$} & \multicolumn{2}{|l|}{$\begin{array}{l}\text { Gruppe II: } 1,2 \pm 0,8 \\
\text { Gruppe III: } 1,3 \pm 0,6\end{array}$} \\
\hline hinterer Kreislauf & 1,3 & & Gruppe III: 1,3 & \\
\hline Aufenthaltsdauer Stroke Unit (in Tagen) & $6,7 \pm 3,8$ & $6,5 \pm 3,7$ & $9,6 \pm 4,1$ & 0,01 \\
\hline vorderer Kreislauf & $6,7 \pm 3,8$ & $6,5 \pm 3,7$ & $9,6 \pm 4,1$ & 0,01 \\
\hline hinterer Kreislauf & $7,1 \pm 2,3$ & $6,3 \pm 2,9$ & $7,9 \pm 3,7$ & 0,03 \\
\hline \multicolumn{5}{|l|}{ Komplikationen (n [\%]) } \\
\hline Re-Infarkt & $3(2)$ & $2(2)$ & $1(5)$ & \\
\hline Gefäßverletzung ACI & $1(1)$ & 0 & $1(5)$ & \\
\hline Pseudoaneurysma & $7(6)$ & $6(6)$ & $1(5)$ & \\
\hline Pneumonie & $19(15)$ & $15(14)$ & $4(21)$ & \\
\hline vorderer Kreislauf & $12(10)$ & $7(6)$ & $5(4)$ & \\
\hline hinterer Kreislauf & $7(6)$ & $3(2)$ & $4(3)$ & \\
\hline Myokardinfarkt & 0 & 0 & 0 & \\
\hline Verstorben & $9(7)$ & $7(7)$ & $2(11)$ & \\
\hline
\end{tabular}

${ }^{1}$ Nicht angegebene Werte lassen sich aufgrund niedriger Fallzahlen nicht berechnen.

schluss im vertebrobasilären Stromgebiet fanden sich signifikant häufiger in der Gruppe II/III ( 12 von 30 vs. 7 von 94 im vorderen Kreislauf; $p<0,001)$. Dennoch musste nur bei einem Patienten mit einem vertebrobasilären Verschluss und primärer Intention zur LA eine sekundäre Intubation erfolgen. Daher scheint es gerechtfertigt, auch bei solchen Patienten primär einen Therapieversuch in LA zu unternehmen, sofern nicht bereits initial zwingende Gründe für eine Intubation vorliegen.

Im vorliegenden Patientenkollektiv bestand kein Unterschied in der Häufigkeit einer Analgosedierung zwischen den beiden Verschlusslokalisationen innerhalb der Gruppe I. Bei Patienten mit einem Verschluss im vorderen Stromgebiet war diese insbesondere dann notwendig, wenn die sprachdominante Hemisphäre betroffen war. In der Regel ist aufgrund der schweren Aphasie eine Kommunikation mit dem Patienten nur eingeschränkt oder nicht möglich. Inwieweit dieser Aspekt das Vorgehen in bisher veröffentlichten Studien beeinflusst hat, wird nicht beschrieben $[10,12]$.

In Analogie zu Jumaa et al. [10] fand sich in der vorliegenden Studie sowohl bei Verschlüssen im vorderen (6,5 $\pm 3,7$ Tage vs. 9,6 $\pm 4,1$ Tage) als auch im hinteren (6,3 $\pm 2,9$ Tage vs. 7,9 $\pm 3,7$ Tage) Kreislauf eine signifikant kürzere Aufenthaltsdauer auf der Stroke Unit für die nicht intubierten Patienten im Vergleich zu den intubierten. Dieser Unterschied kann auch durch einen Selektionsbias bedingt sein, da es plausibel erscheint, dass primär oder sekundär intubierte Patienten wahrscheinlicher einen schwereren bzw. vital bedrohlicheren Schlaganfall erlitten haben und die Gründe für eine Intubation auch den Verlauf nach Intervention und Extubation beeinflussen können. Auch bestand für die Veränderungen in der PCT als morphometrischer Parameter (getrennt nach Gefäßterritorium) kein signifikanter Unterschied zwischen Gruppe I und Gruppe II/III ( $\bullet$ Tab. 1$)$. Somit sind andere Einflussfaktoren für ein erhöhtes Risiko einer Notwendigkeit zur primären oder sekundären ITN zu diskutieren, z. B. Affektion kaudaler Hirnnerven oder zentral bedingte autonome Störungen, die sich nicht in klinischen Skalen oder morphometrischen Analysen widerspiegeln.

Davis et al. konnten in einer retrospektiven, für die Schwere des Schlaganfalls korrigierten Studienpopulation ebenfalls zeigen, dass für die Intervention intubierte Patienten eine signifikant geringere Wahrscheinlichkeit für ein gutes klinisches Outcome haben [24]. In 2 weiteren aktuellen Studien konnte die Korrelation zwischen Intubationsnarkose und schlechterem klinischem Outcome bestätigt werden [25, 26]. Eine mögliche weitere Erklärung hierfür ist, dass sich die Penumbra in Abhängigkeit von der Dauer fehlender Reperfusion zugunsten des Infarktkerns zurückbildet und hieraus ein schlechteres Outcome resultiert [10]. Dieser Effekt wird dadurch noch verstärkt, dass es im Rahmen der Narkoseeinleitung zu unkontrollierten Blutdruckschwankungen mit konsekutiver Veränderung der zerebralen Perfusion kommt kann 
[10, 24, 27, 28]. Ebenso kann das Überstrecken des Kopfes zu einer Alteration der kraniozervikalen Gefäße und der Perfusion führen, vor allem bei vorgeschalteter Stenose [24]. Darüber hinaus kommt es durch den Zeitbedarf für die Narkoseeinleitung zu einer Verzögerung von Interventionsbeginn und konsekutiv damit auch dem Zeitpunkt der Rekanalisation [12, 28]. Dies spiegelt sich im vorliegenden Patientenkollektiv in der signifikant kürzeren Interventionsdauer und „door to recanalization time“ sowohl für einen vertebrobasilären Verschluss als auch einen in der anterioren Zirkulation wider.

Demgegenüber postulieren Brekenfeld et al., dass der Zeitverlust durch die Narkoseeinleitung durch bessere Rekanalisationsraten bei kürzerer Interventionsdauer und geringerer Komplikationsrate ausgeglichen wird [29]. Das Risiko von periprozeduralen Komplikationen durch unkontrollierte Bewegungen bei unkooperativen Patienten wird von verschiedenen Autoren als ein entscheidender Nachteil der Intervention in LA angesehen [11, 29]. Dies konnten wir nicht bestätigen: Die Intervention musste in keinem Fall aufgrund von Bewegungsunruhe abgebrochen werden. In Übereinstimmung mit den Ergebnissen von Jumaa et al. [10] traten auch in unserem Patientenkollektiv periprozedurale Komplikationen häufiger in der Gruppe der intubierten Patienten auf. Kraniozervikale Gefäße sind sehr sensitiv für Scherbewegungen [12]. Daher können bei nicht intubierten Patienten periprozedural neu aufgetretene Schmerzbeschwerden als Warnsignal dienen und zu einer Modifikation des interventionellen Vorgehens führen, um so eine Perforation oder Dissektion zu vermeiden $[12,28]$. In 7 Fällen kam es zu einem technischen Versagen der endovaskulären Therapie. Alle Therapieversager wiesen einen Verschluss im vorderen Kreislauf auf und obwohl sich alle in Gruppe I befanden, waren nicht Bewegungsunruhe, sondern ungünstige anatomische Verhältnisse ursächlich für den Therapieabbruch. Da dieser Faktor nicht durch das anästhesiologische Vorgehen zu beeinflussen ist, wurden diese Patienten aus der weiteren statistischen Analyse ausgeschlossen. Es gibt in den bisher veröffentlichten Studien jedoch keine Angaben, ob diese Patienten a priori aus der weiteren Analyse ausgeschlossen wurden [7, 8, 22, 23, 30].

Die in der Literatur veröffentlichten Rekanalisationsraten (TIMI $2 /$ 3) aus der PROACT-II-Studie [6], der MERCI-Studie sowie den Studien zum Penumbra-System und dem Phenox Clot Retriever reichen von $53-92,4 \%[7,8,22,30]$, sodass die Rekanalisationsraten in der vorliegenden Studie sowohl für das gesamte Patientenkollektiv als auch die einzelnen Subgruppen vergleichbar sind. Die Mortalitäts- und periprozedurale Komplikationsrate liegt mit 7,2\% und 8,8\% (ohne Pneumonie) bzw. 24,1\% mit Pneumonie im unteren Bereich der bisher veröffentlichen Studien [7, 8, 22, 30], insbesondere auch für die intubierten Patienten. Für diese Patientengruppe wird in der Literatur eine Mortalitätsrate von bis zu $60 \%$ beschrieben [31, 32].

Diese Studie hat mehrere Limitationen. Die Hauptlimitation sind die nicht zufällige, durch zentrumsinterne Prozessabläufe nicht beeinflussbare Entscheidung des zuweisenden Notarztes, ob eine primäre Intubation zur Sicherung vitaler Funktionen geboten ist, und die ebenfalls nicht randomisierte fallbezogene Entscheidung des interventionellen Neuroradiologen zu einem bestimmten primären anästhesiologischen Vorgehen. Weitere Störgrößen sind die inhomogenen Gruppengrößen bezüglich des anästhesiologischen Vorgehens und der Verschlusslokalisation, insbesondere ist in der Häufung vertebrobasilärer Insulte in der Gruppe II/III von einem Selektionsbias auszugehen. Die genannten möglichen Störfaktoren können die beobachteten Unterschiede in den Outcomevariablen teilweise erklären oder bedingen, ein kausaler Zusammenhang zwischen anästhesiologischem Vorgehen und Outcome ist somit nicht abzuleiten. Aktuelle Studien zeigen, dass die Rekanalisationsrate durch die Verwendung von Stent-Retrievern gegenüber den in der Studie verwendeten endovaskulären Techniken der mechanischen Rekanalisation noch gesteigert werden kann [5, 30]. Demgegenüber bilden die während des Studienzeitraums verwendeten interventionellen Techniken die während des Studienzeitraumes in der klinischen Routine zur Verfügung stehenden Verfahren $a b$. Weiterhin wurde während des Studienzeitraums keine BridgingTherapie durchgeführt. Durch die ivT vor Beginn der endovaskulären Therapie kann die Zeit zur Narkoseeinleitung bereits für die Schlaganfallbehandlung genutzt werden, jedoch ist das Risiko für Blutungskomplikationen während bzw. durch die Intubation erhöht [24]. Inwieweit das bei Intervention in LA geringere Infarktvolumen im Kontroll-CCT an Tag 5 mit einem verbesserten klinischen Outcome einhergeht, kann angesichts fehlender Daten über den Langzeitverlauf nicht beurteilt werden. Jedoch zeigt sich für die in LA behandelten Patienten in der vorliegenden Studie ein signifikant kürzerer Aufenthalt auf der Stroke Unit, unabhängig von der Verschlusslokalisation. Abschließend wurde eine Vielzahl von Faktoren, die ebenfalls das klinische Outcome beeinflussen können, wie z.B. Begleiterkrankungen und -medikation sowie individuelles Ausmaß der leptomeningealen Kollateralenbildung in den betroffenen Gefäßabschnitten [33] nicht berücksichtigt.

\section{Fazit}

$\nabla$

Lokal rekanalisierende Verfahren bei akut ischämischem Schlaganfall sind unter lokaler Anästhesie im Zugangsbereich ohne erhöhte Komplikationsrate und mit kürzerer Interventionsdauer im Vergleich zur ITN möglich. Eine zeitliche Verzögerung der Therapie durch eine notwendige Intubation kann somit vermieden werden.

\section{Literatur}

1 Rha JH, Saver JL. The impact of recanalization on ischemic stroke outcome: a meta-analysis. Stroke 2007; 38: 967-973

2 Hacke W, Kaste M, Bluhmki E et al. Thrombolysis with alteplase 3 to 4.5 hours after acute ischemic stroke. N Engl J Med 2008; 359: 1317 - 1329

3 Riedel CH, Zimmermann P, Jensen-Kondering $U$ et al. The importance of size: successful recanalization by intravenous thrombolysis in acute anterior stroke depends on thrombus length. Stroke 2011; 42: 1775 - 1777

4 Bhatia R, Hill MD, Shobha $N$ et al. Low rates of acute recanalization with intravenous recombinant tissue plasminogen activator in ischemic stroke: real-world experience and a call for action. Stroke 2010; 41: $2254-2258$

5 Brekenfeld C, Gralla J, Zubler C et al. Mechanische Thrombektomie beim akuten Schlaganfall. Fortschr Röntgenstr 2012; 184: 503 -512

6 Furlan A, Higashida R, Wechsler $L$ et al. Intra-arterial prourokinase for acute ischemic stroke. The PROACT II study: a randomized controlled trial. Prolyse in Acute Cerebral Thromboembolism. JAMA 1999; 282: 2003-2011

7 Penumbra Pivotal Stroke Trial Investigators. The penumbra pivotal stroke trial: safety and effectiveness of a new generation of mechanical devices for clot removal in intracranial large vessel occlusive disease. Stroke 2009; 40: 2761 - 2768

8 Smith WS, Sung G, Saver J et al. Mechanical thrombectomy for acute ischemic stroke: final results of the Multi MERCI trial. Stroke 2008; 39: $1205-1212$

9 Gizewski ER, Weber R, Forsting M. Diagnostik und endivaskuläre Therapie intrakranieller arterieller Stenosen. Fortschr Röntgenstr 2011; 183: $104-111$

10 Jumaa MA, Zhang F, Ruiz-Ares $G$ et al. Comparison of safety and clinical and radiographic outcomes in endovascular acute stroke therapy for 
proximal middle cerebral artery occlusion with intubation and general anesthesia versus the nonintubated state. Stroke 2010; 41: 1180-1184

11 McDonagh DL, Olson DM, Kalia JS et al. Anesthesia and Sedation Practices Among Neurointerventionalists during Acute Ischemic Stroke Endovascular Therapy. Front Neurol 2010; 1: 118

12 Sugg RM, Jackson AS, Holloway W et al. Is mechanical embolectomy performed in nonanesthetized patients effective? Am J Neuroradiol 2010; 31: $1533-1535$

13 Lee CZ, Litt L, Hashimoto T et al. Physiologic monitoring and anesthesia considerations in acute ischemic stroke. J Vasc Interv Radiol 2004; 15 : S13-S19

14 Varma MK, Price K, Jayakrishnan $V$ et al. Anaesthetic considerations for interventional neuroradiology. Br J Anaesth 2007; 99: 75-85

15 Hacke W, Kaste M, Fieschi $C$ et al. Intravenous thrombolysis with recombinant tissue plasminogen activator for acute hemispheric stroke. The European Cooperative Acute Stroke Study (ECASS). JAMA 1995; 274: $1017-1025$

16 Hacke W, Kaste M, Fieschi C et al. Randomised double-blind placebocontrolled trial of thrombolytic therapy with intravenous alteplase in acute ischaemic stroke (ECASS II). Second European-Australasian Acute Stroke Study Investigators. Lancet 1998; 352: 1245-1251

17 Higashida RT, Furlan AJ, Roberts $H$ et al. Trial design and reporting standards for intra-arterial cerebral thrombolysis for acute ischemic stroke. Stroke 2003; 34: e109-e137

18 Gasparotti R, Grassi M, Mardighian D et al. Perfusion CT in patients with acute ischemic stroke treated with intra-arterial thrombolysis: predictive value of infarct core size on clinical outcome. Am J Neuroradiol 2009; 30: $722-727$

19 Lev MH, Segal AZ, Farkas J et al. Utility of perfusion-weighted CT imaging in acute middle cerebral artery stroke treated with intra-arterial thrombolysis: prediction of final infarct volume and clinical outcome. Stroke 2001; 32: 2021-2028

20 Parsons MW, Pepper EM, Chan V et al. Perfusion computed tomography: prediction of final infarct extent and stroke outcome. Ann Neurol 2005; 58: $672-679$

21 Kamalian S, Konstas AA, Maas MB et al. CT Perfusion Mean Transit Time Maps Optimally Distinguish Benign Oligemia from True "At-Risk" Ische- mic Penumbra, but Thresholds Vary by Postprocessing Technique. Am J Neuroradiol 2012; 33: 545-549

22 Bose A, Henkes H, Alfke K et al. The Penumbra System: a mechanical device for the treatment of acute stroke due to thromboembolism. Am J Neuroradiol 2008; 29: 1409-1413

23 The Interventional Management of Stroke (IMS) II Study. Stroke 2007; 38: 2127-2135

24 Davis MJ, Menon BK, Baghirzada LB et al. Anesthetic management and outcome in patients during endovascular therapy for acute stroke. Anesthesiology 2012; 116: 396-405

25 Abou-Chebl A, Lin R, Hussain MS et al. Conscious sedation versus general anesthesia during endovascular therapy for acute anterior circulation stroke: preliminary results from a retrospective, multicenter study. Stroke 2010; 41: 1175 - 1179

26 Nichols C, Carrozzella J, Yeatts $S$ et al. Is periprocedural sedation during acute stroke therapy associated with poorer functional outcomes? J Neurointerv Surg 2010; 2: 67-70

27 Hallevi $H$, Barreto $A D$, Liebeskind DS et al. Identifying patients at high risk for poor outcome after intra-arterial therapy for acute ischemic stroke. Stroke 2009; 40: 1780-1785

28 Molina CA, Selim MH. General or local anesthesia during endovascular procedures: sailing quiet in the darkness or fast under a daylight storm. Stroke 2010; 41: 2720-2721

29 Brekenfeld C, Mattle HP, Schroth G. General is better than local anesthesia during endovascular procedures. Stroke 2010; 41: 2716-2717

30 Henkes $H$, Reinartz J, Lowens $S$ et al. A device for fast mechanical clot retrieval from intracranial arteries (Phenox clot retriever). Neurocrit Care 2006; 5: 134-140

31 Foerch C, Kessler KR, Steckel DA et al. Survival and quality of life outcome after mechanical ventilation in elderly stroke patients. J Neurol Neurosurg Psychiatry 2004; 75: 988 - 993

32 Mayer SA, Copeland D, Bernardini GL et al. Cost and outcome of mechanical ventilation for life-threatening stroke. Stroke 2000; 31: 2346-2353

33 Angermaier A, Langner S, Kirsch $M$ et al. CT-angiographic collateralization predicts final infarct volume after intra-arterial thrombolysis for acute anterior circulation ischemic stroke. Cerebrovasc Dis 2011; 31: $177-184$ 